\title{
Covered Self-Expandable Metal Stents for the Treatment of Benign Biliary Strictures After Liver Transplantation: When and How?
}

\author{
Jong Ho Moon
}

Published online: 17 July 2013

(c) Springer Science+Business Media New York 2013

Benign biliary strictures (BBS) may complicate biliary surgery including liver transplantation (LT) [1]. The firstline management of BBS, including anastomotic strictures after LT, now incorporates endoscopic therapy, mostly consisting of stenting [2]. Although originally designed for endoscopic palliation of malignant biliary strictures, the self-expandable metallic stent (SEMS) is a valid alternative to surgery for BBS. Patients with malignant biliary strictures who undergo treatment with metal stents have a limited life expectancy due to the poor prognosis of the underlying disease. Therefore, for patients with BBS who have a normal life expectancy, the indications for metal stent placement should differ from those applied to malignant biliary strictures.

Metal stents can be divided into uncovered SEMS, partially covered SEMS, and fully covered SEMS (FCSEMS). Non-removable uncovered SEMS cannot be used for BBS due to their limited patency, since reactive hyperplastic tissue may extend through the metal mesh. Partially covered SEMS can be used for BBS to decrease the rate of stent migration; however, the uncovered portion can embed in the bile duct wall. FCSEMS are a good first choice because of their easy removal and long duration of patency due to lack of tissue hyperplasia and implantation into the biliary tree. Since frequent spontaneous stent

\section{J. H. Moon}

Department of Internal Medicine, Digestive Disease Center and Research Institute, Soonchunhyang University School of Medicine, Bucheon/Seoul, Korea

\section{J. H. Moon ( $₫)$}

Digestive Disease Center, Soonchunhyang University Bucheon

Hospital, 1174 Jung-Dong, Wonmi-Ku,

Bucheon 420-767, Korea

e-mail: jhmoon@schmc.ac.kr migration is a major adverse event associated with FCSEMS, an anchoring system such as flared ends or anchoring fins has been developed [3].

In this issue of Digestive Diseases and Sciences, Kahaleh's group reports the retrospectively analyzed outcomes of covered SEMS for 55 patients with anastomotic strictures after LT [4]. Twenty-seven of 55 (49.1\%) patients were treated with plastic stents prior to covered SEMS. The authors applied three different types of covered SEMS: partially covered SEMS (19 patients), FCSEMS with anchoring fins (21 patients), and FCSEMS with flared ends (15 patients). Endoscopic biliary sphincterotomy was performed in all patients. Although the authors endeavored to center the stent within the stricture, success was not universal. All covered SEMS were removed after 3-4 months. The technical success rate was $100 \%$, but the clinical success rate, as measured by stricture resolution, was unsatisfactory (60-74\%). Even after covered SEMS placement, three $(5.5 \%)$ patients eventually required surgery to resolve their biliary strictures. Notably, the stentrelated adverse event rate involving migration and occlusion was 7-24\%. Five patients with early stent occlusion required repeat stenting. Indeed, the clinical success rate of endoscopic stenting with multiple plastic stents is reportedly 87-94\% [5].

The location of the stricture is usually the most proximal aspect of the common hepatic duct in anastomotic strictures after LT, especially after living-related LT recipients, in whom the strictured segment is usually short. To achieve effective stenting with covered SEMS, the stent should be centered within the strictured bile duct segment, which might be difficult to achieve in this clinical setting. Stenting with conventional covered SEMS distal to the stricture of the recipient's native common bile duct can abruptly stretch the duct due to the high radial force developed 
during expansion. Stenting using large-diameter covered SEMS across the papilla facilitates easy reflux of duodenal contents into the biliary tree, clogging the stent and producing biliary inflammation. Duct injuries due to tissue hyperplasia can occur secondary to the presence of flared ends or anchoring fins of FCSEMS in patients with benign strictures [6]. Mahajan et al. [7] implanted an FCSEMS with anchoring fins in 44 patients with BBS. Strictures were resolved in $83 \%$ of patients after placing for a median of 3.3 months. Choledochoscopy in 19 subjects confirmed the presence of mucosal ulceration and bleeding due to trauma from anchoring fins occurring during extraction [7]. Wang et al. [8] demonstrated the absence of migration with the use of this stent in patients with complex bile leakage. Nevertheless, in five of seven patients, mucosal ulceration was demonstrated by choledochoscopy where the fins abutted the duct mucosa [8]. Furthermore, two patients developed a stent-induced stricture distal to the biliary confluence, including one severe stricture that required additional ERCP. De novo strictures have occurred in the bile duct even after the implantation of an FCSEMS lacking flared ends [8], suggesting that the entire bile duct wall covered by a stent can be damaged by the abrupt, high radial force generated by the expansion of the FCSEMS or a disproportionately greater stent diameter relative to the smaller luminal diameter. An FCSEMSinduced second stricture can occur at both stent margins with an incidence of $8-16 \%[6,9]$.

Adverse effects have thus limited the first-line application of FCSEMS in the treatment of BBS. The accompanying paper adds to the hesitation in the use of FCSEMS as a first-line therapy [4]. Therefore, alternatives should be considered. One possibility is plastic stent (PS) placement, which has been used in selected patients with BBS. PS are associated with an overall clinical success rate of 12-100\% [10]. Since their patency is short, and since even two PS cannot create enough radial force to resolve a tight stricture, their long-term outcomes are not satisfactory despite their high early success rate. Multiple PS placement has been proposed to generate adequate radial force to resolve strictures with a reported long-term success rate $>88 \%[5$, $10]$, at the cost of the need for repeated endoscopic procedures. To date, however, consensus regarding multiple PS placement is lacking. No controlled prospective studies have compared the effectiveness of multiple PS with FCSEMS. There is no definitive evidence supporting the hypothesis that FCSEMS is superior to PS for patients with anastomotic strictures following LT [11, 12]. Since FCSEMS placement can be performed in one step, it is logical to first place a PS for their early efficacy, followed by FCSEMS for more durable results. Despite the unsatisfactory stricture resolution rate in this study, the long-term effectiveness of FCSEMS placement has been reported in many studies $[11,12]$. Thus, FCSEMS can be used to effectively manage selected patients with refractory BBS before surgical intervention.

Newly designed FCSEMS have been developed to overcome the disadvantages of the current FCSEMS. A modified FCSEMS, placed intraductally through the strictured segment to minimize stent-induced complications has been reported [13]. In our experience, intraductal placement of a short FCSEMS for a BBS in patients who had undergone living-related LT was not associated with stent-induced bile duct injury, including de novo stricture formation or cholangitis. Intraductally located FCSEMS can be removed endoscopically after 3 months without complication. Although further studies are needed to demonstrate the efficacy of this technique, the intraductal insertion of a short FCSEMS appears to have promise based on our preliminary results and the reported experience of others.

In conclusion, the application of SEMS can be reserved for unsuccessful cases of PS placement, although promising evidence has been introduced recently to support FCSEMS for anastomotic strictures after LT. Further studies with existing devices or the development of new FCSEMS are needed before recommending the routine use of FCSEMS in this clinical setting.

Conflict of interest None.

\section{References}

1. Kahaleh M, Behm B, Clarke BW, et al. Temporary placement of covered self-expandable metal stents in benign biliary strictures: a new paradigm? (with video). Gastrointest Endosc. 2008;67: 446-454.

2. Pasha SF, Harrison ME, Das A, et al. Endoscopic treatment of anastomotic biliary strictures after deceased donor liver transplantation: outcomes after maximal stent therapy. Gastrointest Endosc. 2007;66:44-51.

3. Park JK, Moon JH, Choi HJ, et al. Anchoring of a fully covered self-expandable metal stent with a $5 \mathrm{~F}$ double-pigtail plastic stent to prevent migration in the management of benign biliary strictures. Am J Gastroenterol. 2011;106:1761-1765.

4. Cerecedo-Rodriguez J, Phillips M, Figueroa-Barojas $\mathrm{P}$, et al. Self-expandable metal stents for anastomotic stricture following liver transplant. Dig Dis Sci. (Epub ahead of print). doi:10.1007/ s10620-013-2703-0.

5. Costamagna G, Pandolfi M, Mutignani M, Spada C, Perri V. Long-term results of endoscopic management of postoperative bile duct strictures with increasing numbers of stents. Gastrointest Endosc. 2001;54:162-168.

6. Moon SH, Kim MH, Park DH, et al. Modified fully covered selfexpandable metal stents with antimigration features for benign pancreatic-duct strictures in advanced chronic pancreatitis, with a focus on the safety profile and reducing migration. Gastrointest Endosc. 2010;72:86-91.

7. Mahajan A, Ho H, Sauer B, et al. Temporary placement of fully covered self-expandable metal stents in benign biliary strictures: midterm evaluation (with video). Gastrointest Endosc. 2009; 70:303-309. 
8. Wang AY, Ellen K, Berg CL, Schmitt TM, Kahaleh M. Fully covered self-expandable metallic stents in the management of complex biliary leaks: preliminary data-a case series. Endoscopy. 2009;41:781-786.

9. Phillips MS, Bonatti H, Sauer BG, et al. Elevated stricture rate following the use of fully covered self-expandable metal biliary stents for biliary leaks following liver transplantation. Endoscopy. 2011;43:512-517.

10. van Boeckel PG, Vleggaar FP, Siersema PD. Plastic or metal stents for benign extrahepatic biliary strictures: a systematic review. BMC Gastroenterol. 2009;9:96.

11. Kao D, Zepeda-Gomez S, Tandon P, Bain VG. Managing the post-liver transplantation anastomotic biliary stricture: multiple plastic versus metal stents: a systematic review. Gastrointest Endosc. 2013;77:679-691.

12. Kaffes AJ, Liu K. Fully covered self-expandable metal stents for treatment of benign biliary strictures. Gastrointest Endosc. 2013;78:13-21.

13. Moon JH, Choi HJ, Koo HC, et al. Feasibility of placing a modified fully covered self-expandable metal stent above the papilla to minimize stent-induced bile duct injury in patients with refractory benign biliary strictures (with videos). Gastrointest Endosc. 2012;75:1080-1085. 